\title{
DAS MEXIKANISCHE MILITÄR: EIN LATEINAMERIKANISCHER SONDERFALL?
}

\author{
Von VOlKer G. LeHr
}

Daß die Militärs die Richtlinien der Politik diktieren, ist in Lateinamerika der Normalfall. Als eine der wenigen Ausnahmen gilt Mexiko. Dort vereinigte 1929 Präsident Calles die an der Volksrevolution (1910-1917) beteiligten Kräfte in der sog. offiziellen Partei, um den „,caudillismo“ durch eine institutionenbezogene, zivile Politik abzulösen. Nach zweifacher Umbenennung heißt die Partei seit 1946 ,Partido Revolucionario Institucional“ (PRI). Während der Parteigründung 1929 rebellierten einige Generäle, aber die Erhebung wurde rasch niedergeschlagen. Es handelte sich um den letzten militärischen Versuch, die Macht zu ergreifen. Ein mit enormer Machtfülle ausgestatteter, aber nicht wiederwählbarer Präsident und der PRI, in den Arbeiter, Bauern und Mittelstand als Sektoren inkorporiert sind, sind die beiden herausragenden Charakteristika des autoritären mexikanischen Regimes, das es hinter einer formaldemokratischen Fassade verstanden hat, die Opposition, der ein begrenzter Spielraum zugestanden wird, geschickt zu mediatisieren. Unter Beibehaltung einer revolutionären Rhetorik und einer ,,Massenmobilisierung unter gleichzeitiger Massenkontrolle" ${ }^{\text {"1 }}$ beschleunigte das PRI-Regime nach 1940 eine kapitalistische Modernisierung, die - bei einer gleichzeitigen Zunahme regionaler und sozialer Disparitäten - zu einem enormen Wirtschaftswachstum führte. Die politische „Erfolgsbilanz“ des PRI-Regimes ist beachtlich: Es stellt seit über 50 Jahren alle nationalen und bundesstaatlichen Regierungen und erreichte eine nicht nur in Lateinamerika ungewöhnliche Stabilität; heute ist es das älteste autoritäre Regime der Welt ${ }^{2}$. Avila Camacho, der letzte mexikanische Präsident, der aus den Streitkräften der Revolution hervorgegangen war und den Generalsrang trug, sorgte dafür, daß mit Alemán ein ziviler Politiker seine Nachfolge antrat - ein Zeichen für die Konsolidierung des Regimes und die Unterordnung der Armee unter die politische Führung. Damit ist Mexiko - abgesehen von Costa Rica, wo nach dem Bürgerkrieg von 1948 das Militär durch Verfassungsreform sogar abgeschafft wurde - das lateinamerikanische Land, welches am längsten ununterbrochen von zivilen Staatschefs regiert wird. Hingegen regierten noch in den 50er Jahren Militärdiktatoren in den beiden Ländern, die heute neben Costa Rica und Mexiko als die Beispiele ziviler Herrschaft in Lateinamerika gelten: In Venezuela übte 1948-1958 der Gen. Pérez Jiménez die Macht aus, und von 1953-1957 herrschte Gen. Rojas Pinilla in Kolumbien, wo auch heute noch der militärische Einfluß deutlicher als in den vorgenannten Ländern zu spüren ist.

\section{Mexikanische Militäraufwendungen im lateinamerikanischen Vergleich.}

Die mexikanischen Streitkräfte sind für die Größe des Landes, d. h. hinsichtlich seiner Einwohnerzahl und seiner Wirtschaftskraft, relativ gering ausgebaut - weit weniger, als dies sonst in Lateinamerika üblich ist (siehe die nachstehenden Tabellen auf S. 79 und 80). Während 1977 in ganz Lateinamerika 4,27 Soldaten auf 1000 Einwohnern kamen, so waren es in Mexiko nur 1,57 Soldaten (Rang 20 unter 25 lateinamerikanischen Staaten). Nur in fünf

1 Tobler, Hans, W. in: Mols, Manfred und Hans W. Tobler: Mexiko. Die Institutionalisierte Revolution. Köln 1976, S. 32.

2 Ich folge hier dem von Juan Linz entwickelten Autoritarismuskonzept, s. ders.: Totalitarian and Authoritarian Regimes. In: Greenstein, Fred I. u. Nelson W. Polsby (Hrsg.): Handbook of Political Science. Vol. 3. Macropolitical Theory. Reading, Mass. 1975, S. $175-411$. 
Kleinstaaten waren es noch weniger: in Costa Rica, das nur eine Bürgerwehr unterhält, in Haiti, wo sich die Diktatur der Familie Duvalier auf die paramilitärischen Banden der sogenannten Tontons Macoutes stützt, und in den ehemaligen britischen Kolonien Trinidad und Tobago, Barbados und Jamaica. Von dem lateinamerikanischen BSP entfielen 1977 1,50 \% auf Militärausgaben; in Mexiko waren es mit 0,73 \% noch nicht einmal halb soviel (Rang 19 unter 24 Staaten). Einen geringeren Anteil hatten die Militärausgaben nur in Kolumbien, Panama, Trinidad und Tobago, Barbados und Costa Rica.

In absoluten Ziffern gemessen rangieren die mexikanischen Streitkräfte mit 100000 Berufssoldaten allerdings erheblich weiter vorne: auf dem 6. Rang hinter Brasilien, Kuba, Argentinien, Peru und Chile. Die Militärausgaben waren 1977 nur in Brasilien, Peru, Argentinien und Venezuela höher als in Mexiko (Für Kuba gibt es für 1977 keine Angaben). In relativen Zahlen sind die mexikanischen Militäraufwendungen im unteren Drittel der lateinamerikanischen Staaten angesiedelt, in absoluten Zahlen jedoch im oberen Drittel. Der Anteil der Militärausgaben an den gesamten Ausgaben der Bundesregierung ist von der Amtszeit Carranzas (1917-20) bis zur Amtszeit Echeverrías (1970-76) stark gesunken - von 55,2 \% auf $5,8 \%^{3}$. In den letzten Jahren sind die Ausgaben allerdings relativ konstant geblieben, was sich insbesondere dann zeigt, wenn man sie als BSP-Anteil mißt; seit 1961 liegt er recht beständig bei 0,7 \%. Ebenfalls ist die Mannschaftsstärke der Armee dem Bevölkerungswachstum stets angeglichen worden (1961: 1,61 Soldaten auf $1000 \mathrm{EW}$; 1977: 1,57 Soldaten) ${ }^{4}$.

\section{Die politische Neutralisierung des mexikanischen Militärs.}

Daß die mexikanische Politik nach der Revolution allmählich demilitarisiert wurde, zeigt sich vor allem, wenn man die Herkunft der Spitzenpolitiker in Augenschein nimmt. Von 1917 bis 1946 waren die Staatspräsidenten überwiegend Generäle gewesen; seit 1946 waren dann allerdings die Präsidenten ausnahmslos Zivilisten. Die Anzahl der Militärs, die zu Beginn der 20er Jahre mehrheitlich im Kabinett vertreten waren, ist rasch zurückgegangen. Seit 1946 zählen von den Ministerien nur noch Verteidigungs- und Marineministerium zum festen Besitzstand der Armee. Es kommt jedoch noch die Oberbehörde der Rüstungsindustrie hinzu; ferner werden traditionellerweise Armeeoffiziere an die Spitze der verschiedenen Polizeidienste berufen. Im Sicherheitsbereich haben die Militärs ihre.Stellung behaupten können. Von den ersten 19 Vorsitzenden, die die Revolutionspartei von 1929 bis 1964 hatte, waren elf Generäle. Im Anschluß daran ist jedoch der PRI-Vorsitz bisher, d. h. bis 1980, von acht zivilen Politikern eingenommen worden. Nach 1940 ist ebenfalls der recht hohe Anteil von Offizieren, die öffentliche Wahlämter innehatten, zurückgegangen. Vor allem unter den Gouverneuren und Senatoren hatte es eine Reihe von Offizieren gegeben (die unteren Wahlämter dürften ihren militärischen Rängen weniger entsprochen haben). 1979 waren von den 31 Gouverneuren 3, von den 64 Senatoren 2 und von den 237 Abgeordneten 1 Militärs. Auf allen Ämterniveaus (Ausnahme Sicherheitsbereich!) läßt sich mit zunehmender Konsolidierung des Regimes eine Demilitarisierung der mexikanischen Politik feststellen.

Die ersten Maßnahmen zur Neutralisierung des militärischen Machtfaktors waren 1924 die Einteilung des Landes in 33 (später 35) Militärzonen, die in der überwiegenden Anzahl mit

3 Für die Jahre 1917-1963 s. Wilkie, James W.: The Mexican Revolution: Federal Expenditure and Social Change since 1910. Berkeley, Cal. 1970, S. 108 f. u. für die Jahre 1968-1977: U. S. Arms Control and Disarmament Agency: World Military Expenditures and Arms Transfers 1968-1977. Washington, D. C. 1979.

4 U. S. Arms Control . . . World Military Expenditures 1971. Washington, D. C. 1972, S. 27, 31 u. 35 und U. S. Arms Control . . .: World Military Expenditures . . 1968-1977. Washington, D. C. 1979, S. 53 u. 96. 
den Bundesstaaten deckungsgleich sind, und die Verfügung, daß die Kommandanten der Zonen - die vom Staatspräsidenten selber ernannt werden ${ }^{5}$ - periodisch versetzt werden. Damit sollte die Möglichkeit eingeschränkt werden, daß die Truppe zu den regionalen Befehlshabern enge persönliche Treueverhältnisse entwickelt. Es handelte sich um eine Maßnahme gegen den auf regionalen Machtbasen beruhenden ,,caudillismo“ der Heerführer einerseits und der politischen Gouverneure andererseits ${ }^{6}$. Die von Zone zu Zone rotierenden Kommandanten, die nur der nationalen Exekutive unterstellt sind, dienen nämlich gleichzeitig zur Kontrolle der Gouverneure. Die Devise „Divide et impera“ dürfte auch bei den darauffolgenden Maßnahmen entscheidungsrelevant gewesen sein. Kurz nachdem Avila Camacho, der unter Präsident Gen. Cárdenas (1934-40) Verteidigungsminister gewesen war, 1939 als offizieller Präsidentschaftskandidat nominiert worden war, wurde der Marinebereich aus dem Verteidigungsministerium ausgegliedert und als Oberbehörde direkt dem Präsidenten unterstellt. Erstmals hatten Heer und Marine erst im Präsidenten, der Oberbefehlshaber aller Streitkräfte ist, ihren gemeinsamen Vorgesetżten. Ein Jahr später wurde das ,,Departamento de la Marina“"zum Ministerium, zur ,Secretaría de Marina“ erhoben. Damit standen zwei Posten mit Ministerrang zur Kooptation der Militärs zur Verfügung. Wenige Tage nach seinem Amtsantritt 1940 ließ Avila Camacho den ,,sector militar" der Revolutionspartei, in den alle Soldaten ohne Ausnahme inkorporiert waren, auflösen, um den Streitkräften als Verband ihren politischen Charakter zu nehmen und sie aus dem Wahlgeschehen herauszu-

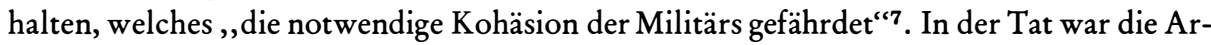
mee durch den heftig geführten Präsidentschaftswahlkampf des Jahres 1940 Spannungen ausgesetzt worden. Zwar hatte Avila Camacho, der ehemalige Verteidigungsminister, die Mehrheit der Armee hinter sich; aber einige ältere Militärs sollen ihn nicht akzeptiert haben, da er seine Karriere nicht auf dem Schlachtfeld der Revolution errungen, sondern sich in der politisch-militärischen Bürokratie hochgedient hatte. Sein Gegenkandidat, Gen. Almazán, war hingegen aktiv an den revolutionären Kämpfen beteiligt gewesen (was sich freilich nicht auf seine politische Position, die konservativer als diejenige Avila Camachos war, auswirkte). Auch wenn Almazán nach seiner Wahlniederlage, der von den offiziellen Stellen zumindest nachgeholfen worden war, davon Abstand nahm, mit militärischer Gewalt ins Präsidentenamt zu gelangen, so war doch deutlich geworden, daß die Politisierung die Einigkeit der Armee beeinträchtigt hatte und sie von ihren eigentlichen militärischen Aufgaben abgelenkt hatte. Auch angesichts des entfachten 2 . Weltkriegs erschien es geboten, die Streitkräfte von den innenpolitischen Querelen zu entfernen und sie auf ihre eigentlichen Kompetenzen zu konzentrieren. Es wurde allerdings den Militärs freigestellt, als Einzelpersonen über den Mittelstandssektor der Revolutionspartei beizutreten und politische Karrieren zu machen. 1942 ließ Avila Camacho dann den Präsidentenstab ins Leben rufen, dem die Präsidentengarde unterstellt ist. Dadurch wurde - zumeist jüngeren - Offizieren die Gelegenheit geboten, sich durch loyalen und umsichtigen Dienst die Anerkennung des Obersten Befehlshabers der Streitkräfte zu erwerben. Sie sind dem Präsidenten unmittelbar verpflichtet und üben ihre Tätigkeit in dessen engster Umgebung aus.

Der Nachfolger Avila Camachos, Miguel Alemán, nahm 1947 die Rüstungsindustrie aus dem Zuständigkeitsbereich des Verteidigungsministeriums heraus und schuf eine dem Präsidenten direkt unterstellte Oberbehörde, das „Departamento de la Industria Militar“. So

5 Gemäß Verfassungsartikel 89 werden alle Offiziere unmittelbar vom Präsidenten ernannt; bei den höchsten Rängen - vom Oberst aufwärts - bedarf es der Zustimmung des Senats.

6 Vgl. Lozoya, Jorge, A.: El ejercito mexicano. México, D. F. 1976, S. 79 f. u. 105 ff.

7 Siehe das Dekret von Avila Camacho in: Osorio Marban, Miguel: El Partido de la Revolución Mexicana. México, D. F. 1970, Bd. 2, S. 793. 
sind die verschiedenen Kommandos - Verteidigungsministerium (das neben dem Heer auch für die Luftwaffe zuständig ist, die jedoch erst neuerdings an Bedeutung gewinnt), Marineministerium, Präsidentenstab (mit Präsidentengarde) und Departement für Rüstungsindustrie - erst in den Händen des Staatspräsidenten vereint. Zur politischen Neutralisierung der Armee sollen auch die Gratifikationen für Offiziere und Soldaten sowie deren Familien dienen. Es existiert ein ausgedehntes Netz sozialer Sicherheit. Die Armeeangehörigen haben Eigenheime, eigene Läden, in denen die gängigen Verbrauchsgüter günstig gekauft werden können, eigene Hospitäler, Schulen und Hochschulen. Es wird die Meinung vertreten, daß diese Leistungen die Loyalität des Militärs gegenüber dem Regime stützen ${ }^{8}$. Indes gilt es zu bedenken, daß die damit einhergehende Isolierung von der zivilen Gesellschaft auch der Entwicklung eines unter Umständen bedrohlichen castrensischen Geistes förderlich ist. Ubbrigens, warum sollten die Gratifikationen unter einer Militärregierung geringer ausfallen? Ebenfalls zur Kooptation der Militärs gehörte es, daß während der Präsidentschaft Echeverrías (1970-76) im Süden der Hauptstadt ein gigantisches und futuristisch anmutendes ,,Colegio Militar" errichtet wurde. Auch in diesem Fall mag es Langzeiteffekte geben, die mit den ursprünglichen Absichten nicht mehr übereinstimmen.

\section{Aufgaben und Aktivitäten des mexikanischen Militärs.}

Die Verfassung überträgt den mexikanischen Streitkräften explizit nicht nur eine externe, sondern auch eine interne Funktion. Der Präsident verfügt gemäß Verfassungsartikel 89, Abs. VI, über die Gesamtheit der Streitkräfte ,,zur inneren Sicherheit und der äußeren Verteidigung der Föderation“. In der mexikanischen Militärdoktrin hat die Gewährleistung der inneren ,,Ruhe und Ordnung“ 1952 die Verteidigung gegenüber Angriffen von außen vom ersten Rang unter den Aufgaben verdrängt ${ }^{9}$. Da Mexikos südliche Nachbarn, Guatemala und Belize, keine ernsthaften Gegner wären und gegen die USA, den Koloß im Norden, ohnehin jegliche militärische Anstrengung vergebens wäre, wird der zu Beginn des 2. Weltkriegs eingeführte obligatorische Wehrdienst, der sich auf knapp 1 Jahr erstreckt, äußerst lasch gehandhabt. Der Regierung bietet er vor allem eine Gelegenheit, die männliche Bevölkerung über 18 Jahre zu registrieren.

Wie in den anderen lateinamerikanischen Staaten bewegen sich die Aktivitäten der mexikanischen Armee zwischen ,,civic action " und ,,counterinsurgency“. Sie reichen von Alphabetisierungskampagnen unter den Wehrpflichtigen, Straßenbau, Wasserversorgungsprogrammen, Aufforstung, medizinischer Versorgung usw. über die Bekämpfung des Drogenanbaus und -handels, die Sicherung der öffentlichen Ordnung an Wahltagen bis hin zu repressiven und oft blutigen Einsätzen, zur gewaltsamen Auflösung von Streiks, zur Niederwerfung von Studentenunruhen, zur Unterdrückung von Unruhen unter der Landbevölkerung und zur Bekämpfung der Guerilla ${ }^{10}$. Wenn Kooptationsversuche erfolglos bleiben, ist damit zu rechnen, daß das Militär eingesetzt wird, um Widerstand unter Einsatz von Gewalt zu brechen. Insbesondere in entlegenen ländlichen Gebieten ist die Kontrolle des Heeres äußerst intensiv. In dieser Hinsicht ist die mexikanische Armee kaum weniger aktiv als ihre ,,counterparts" in anderen lateinamerikanischen Ländern"11. Seit der 2. Hälfte der 60er Jahre hat

8 Vgl. Lozoya, Jorge, A., op. cit., S. 109.

9 Ibid., S. $119 \mathrm{f}$.

10 Beispiele finden sich bei Boils, Guillermo: Los militares y la política en México (1915-1974). México, D. F. 1975, S. 115 ff. u. bei Ronfeldt, David F.: The Mexican Army and Political Order since 1940. In: Wilkie, James W. et al.: Contemporary Mexico. Berkeley 1976, S. $317 \mathrm{ff}$.

11 Vgl. Ronfeldt, David F., op. cit., S. 324 
das Militär in offenen und zunehmend verdeckten Aktionen die verschiedenen linken Guerillagruppen - Movimiento de Acción Revolucionaria, Frento Urbano Zapatista, Fuerzas Revolucionarias Armadas del Pueblo, Liga Comunista 23 de Septiembre, Unión del Pueblo usw. - bekämpft. Die Maßnahmen der Militärs werden nicht nur oft außerhalb der Gesetze und der Kontrolle durch die Justiz entzogen durchgeführt, sondern sie treffen häufig auch Personen, die in keiner Beziehung zur Guerilla stehen. 1979 wurde z. B. der Jurist Bautista Rosas, der 17 Jahre lang Offizier gewesen war, vermutlich von Sicherheitskräften entführt, nachdem die Wochenzeitschrift Proceso Auszüge aus dessen militärkritischer Examensarbeit, in der er unter anderem auf die repressiven Aktivitäten des Heeres eingegangen war, veröffentlicht hatte. Als sich die Proteste aus der Offentlichkeit häuften, wurde Bautista nach 6 Tagen, in denen er in einem geheimen Gefängnis (zusammen mit anderen Gefangenen) festgehalten und über seine Studie verhört worden war, wieder freigelassen ${ }^{12}$. Immer wieder wird von Entführungen, Geheimgefängnissen und Folterverhören berichtet. Trotz der Freilassung von über 400 politischen Gefangenen 1977 und weiterer 250 politischer Gefangenen aufgrund des Amnestiegesetzes von 1978 gibt die Situation der Menschenrechte in Mexiko, das international als großzügiges Asylland Ansehen genießt, weiterhin zu Sorge Anlaß. In seinem Jahresbericht 1979 bezweifelt Amnesty International die Behauptung des mexikanischen Generalstaatsanwaltes Flores Sánchez, ,,daß die Folter in Mexiko nur begrenzt und in geringem Maße angewendet werde, da die Organisation viele fundierte Berichte erhalten hat über die Anwendung der Folter, die ausdrücklich von der Verfassung verboten wird"13. Als berüchtigtes Geheimgefängnis gilt das am Rande der Hauptstadt gelegene Militärlager Nr. 1. Zu den ,,Verschwundenen“ erklärte Flores Sánchez auf einer internationalen Pressekonferenz am 24. Januar 1979, ,,daß von den 314 den Behörden bekannten Fällen 154 Personen von Polizei oder Armee getötet worden seien (d. h. in Gefechten mit den Sicherheitskräften; V. L.), 89 sich versteckt hielten und weitere 58 aufgrund von Gewalttätigkeiten zwischen rivalisierenden Guerillagruppen zu Tode gekommen seien"14. Obwohl die Regierung stets die Existenz der sog. ,,Brigada Blanca“, einer inoffiziellen Einheit zur Bekämpfung subversiver Aktivitäten, geleugnet hat, gibt es Hinweise, die an den amtlichen Verlautbarungen ernsthafte $Z$ weifel aufkommen lassen.

Proceso veröffentlichte im Januar 1980 verschiedene interne amtliche Schreiben der Sicherheitskräfte, in denen die „Brigada Blanca“ explizit genannt wird ${ }^{15}$. Die „Brigada Blanca“, deren Mitglieder vermutlich der Armee und den verschiedenen Polizeidiensten entstammen, dürfte das mexikanische Pendant zu den brasilianischen Todesschwadronen, der argentinischen ,Triple A“ (Argentinische Antikommunistische Allianz), der guatemaltekischen „Mano Blanco“ (Weiße Hand) und zur salvadorenischen „,Organización Democrática Nacional - ORDEN “ sein. Die „,Brigada Blanca“ soll 1976 gegründet worden sein und ist seit 1977 unter diesem Namen in der Offentlichkeit bekannt.

Bislang waren und sind die im wesentlichen vom Militär getragenen und geführten Sicherheitskräfte ein Ordnungs- und Repressionsinstrument in der Hand der Regierung; allerdings sind gefährliche Verselbständigungsprozesse seitens der Sicherheitskräfte, die oft außerhalb der Verfassugsnormen und der rechtlich-institutionellen Verfahren agieren, nicht zu übersehen.

12 Siehe Proceso Nr. 111 v. 18. 12. 1978, S. 18-21, Nr. 113 v. 1. 1. 1979, S. 29, u. Nr. 114 v. 8. 1. 1979, S. 14 f.

13 Amnesty International: Jahresbericht 1979. Frankfurt 1980, S. 122.

14 Ibid., S. 121 f.; vgl. Proceso Nr. 117 v. 29. 1. 1979, S. 16 f.

15 Proceso Nr. 166 v. 17. 1. 1980, S. 6-10. 


\section{Remilitarisierung - eine Möglichkeit Mexikos politischer Zukunft?}

Zwar ist - wie aufgezeigt wurde - die mexikanische Politik mit Ausnahme des Sicherheitsbereiches weitgehend demilitarisiert worden, aber dies sagt angesichts lateinamerikanischer Erfahrungen wenig über künftige Entwicklungen aus. Auch die Streitkräfte Uruguays und Chiles galten lange Zeit als entpolitisiert und als ausgesprochen loyal gegenüber den zivilen Institutionen ihrer Länder. Nichtsdestoweniger übernahm das uruguayische Militär in den 70er Jahren allmählich - zunächst indirekt, dann offen regierend - die Macht; in Chile putschte 1973 das Militär gegen Allende und ergriff die Macht.

Welches Selbstverständnis in dem mexikanischen Offizierskorps vorherrscht, ist ungewiß und müßte eruiert werden. Es wäre ebenfalls erforderlich zu wissen, was im neuen ,,Colegio Militar" auf dem Lehrplan derKadetten steht. Zu denken geben sollte jedenfalls die folgende Passage eines Interviews, das der Publizist Ricardo Garibay 1978 mit dem Gouverneur des Bundesstaates Oaxaca, General Jiménez Ruiz, führte:

,,- Sollen die Militärs an die Macht zurückkehren? Werden sie zurückkehren?

- . . Von der Verfassung her gibt es kein Problem.

- Wäre es angebracht für Mexiko, daß die Militärs an die Macht zurückkehren?

- Wir Militärs haben drei Pflichten von heiliger Ordnung: über die territoriale Integrität der Republik zu wachen, die friedliche Ausübung der Institutionen aufrechtzuerhalten und die Souveränität des Staates und der Nation zu beschützen.

- Wäre es angebracht für Mexiko, daß die Militärs an die Macht zurückkehren?

- Antworten Sie mir bitte, Freund Caribay; wenn Aufrichtigkeit, Gefühl und Bereitschaft zu dienen vorhanden sind, was bedeutet dann die Herkunft (der Regierenden; V.L.)?“ 16.

Die ausweichenden Antworten des Generals und Gouverneurs sind ein Indiz dafür, daß man in militärischen Kreisen politische Führungsaspirationen zumindest nicht völlig aufgegeben hat. Wenngleich man - insgesamt gesehen - gegenwärtig das mexikanische Militär als einen der politischen Führung untergeordneten Bestandteil des Herrschaftsapparates des PRI-Regimes betrachten muß, so lassen doch gewisse Umstände eine Rückkehr des mexikanischen Militärs an die Macht nicht zwingend, aber möglich erscheinen:

1. Die Generation der Offiziere, deren Identifikation mit dem Regime auf der Teilnahme an der bewaffneten Revolution herrührt, ist längst pensioniert bzw. verstorben. Einer der letzten Vertreter der alten Garde war der inzwischen auch verstorbene Verteidigungsminister Echeverrías, General Cuenca Díaz.

2. Die nachfolgenden Generationen der Offiziere, die z. T. zusammen mit Offizieren anderer Länder Lateinamerikas in den USA oder in der Kanalzone von Panama geschult worden sind, blicken möglicherweise mit Neid auf die Machtfülle anderer lateinamerikanischer Armeen, die zudem in relativen Zahlen hinsichtlich der Militärauf wendungen bisher weit günstiger als die mexikanischen Streitkräfte dastehen. Es ist aber damit zu rechnen, daß die mexikanischen Militäraufwendungen künftig stärker als bisher ansteigen, damit das Land im Ernstfall bei der Verteidigung des neuen Petroleumreichtums nicht auf ausländische Hilfe angewiesen ist. Die politische Machtfülle ihrer lateinamerikanischen Kollegen könnte indes - um es einmal etwas prononciert zu formulieren - bei den mexikanischen Offizieren weiterhin Minderwertigkeitskomplexe verursachen.

16 Proceso Nr. 68 v. 20. 2. 1978 , S. 17. 
3. Die Aufgabe, die Erdölvorkommen zu sichern, verschafft dem Militär vermutlich mehr Einfluß und Gewicht.

4. Die Ereignisse gegen Ende der Amtszeit Echeverrías dürften etwaige Machtaspirationen verstärkt haben. Die Wirtschaftskrise des Jahres 1976 führte in der Bevölkerung zu einem enormen Vertrauensschwund gegenüber den politischen Institutionen. Der Vertrauensschwund äußerte sich unter anderem in der Bereitwilligeit, Gerüchten über einen Militärputsch Glauben zu schenken. 1976 könnten die Militärs durchaus den Eindruck gewonnen haben, daß ein Staatsstreich bei einem großen Teil der Bevölkerung auf Verständnis gestoßen wäre ${ }^{17}$.

5. Die seit Jahren zu beobachtende Verschärfung der sozialen und regionalen Disparitäten, die andauernde Auslandsabhängigkeit und die raschen sozioökonomischen Veränderungen im Gefolge des Erdölbooms dürften das Land erheblichen Spannungen aussetzen.

6. Bereits jetzt sprechen die Wahlergebnisse für einen deutlichen Legitimitätsverfall des herrschenden Systems. Die verschiedenen politischen Reformen (1962/63, 1972/73 und 1977), die nur vordergründig den Spielraum der Oppositionsparteien erweiterten, letztlich aber einer Perpetuierung der Herrschaftsverhältnisse dienten, haben es nicht vermocht zu verhindern, daß die politische Entfremdung der Wahlbürger zugenommen hat ${ }^{18}$. In den neun Abgeordnetenwahlen seit 1955 ist der Anteil der registrierten Wahlbürger, die für den PRI stimmen, erheblich gesunken - von 62,2\% (1955) auf 34,8\% (1979), ohne daß die Oppositionsparteien auch nur in annäherndem Maße hinzugewannen. Sie erhöhten ihren Stimmenanteil unter den registrierten Wahlbürgern gerade um 5,1 \% - von 7,0 \% (1955) auf 12,1\% (1979), ein Ergebnis, das sie bereits 1973 erreicht hatten. Stark gestiegen ist hingegen der Anteil derer, die gar keiner Partei ihre Stimme gaben, d. h. die entweder den Urnen fernblieben oder ihre Stimmzettel annullierten. Er hat von 30,8 \% (1955) auf 53,1 \% (1979) zugenommen. Selbst wenn man berücksichtigt, daß in den Jahren, in denen auch der Präsident gewählt wird, normalerweise die Beteiligung an den Abgeordnetenwahlen und der PRI-Stimmenanteil etwas höher liegen, würden - bei einer Fortsetzung der zwischen 1955 und 1979 beobachteten Trends - im Jahr 2000 nur noch $20 \%$ der registrierten Wahlbürger für den PRI, weitere $15 \%$ für die Oppositionsparteien, aber $65 \%$ für gar keine Partei stimmen ${ }^{19}$. Auch wenn sich in der Realität das Legitimitätsvakuum nicht wie im statistischen Szenario vergrößern sollte, so hat es bereits in der Gegenwart bedenkliche Ausmaße angenommen. Eine Zuspitzung der wirtschaftlich-sozialen Krisen und ein anhaltendes oder sich gar ausweitendes Legitimitätsvakuum könnten zu einer Situation führen, in der das Militär nicht mehr bereit wäre, das PRI-Regime (und die mit ihm assoziierte Korruption, Bereicherung und administrative Ineffizienz) zu stützen, sondern es anstrebte, in eigener Verantwortung die Macht auszuüben und die Ordnung zu gewährleisten. Es ist fraglich, ob in einer schweren Krisensituation das von den PRI- Regierungen gegenüber dem Militär befolgte „,Divide et impera"-Prinzip ebenso wie die Gratifikationen-Politik noch wirkungsvoll wäre. Es ist ebenso fraglich, ob der Apparat des PRI im Ernstfall noch einen kampffähigen Machtfaktor

17 Hingegen hatte die Armee 1968, als angesichts der blutigen Niederwerfung der Studentenbewegung ebenfalls Gerüchte über eine militärische Machtergreifung zirkulierten, aufgrund des von ihr durchgeführten Gemetzels von Tlatelolco, bei dem schätzungsweise 200 Personen getötet wurden (New York Times v. 9. 7. 1970, S. 12), die Volksmeinung wahrscheinlich gegen sich.

$18 \mathrm{Vgl}$. zur politischen Reform von 1977 und den darauffolgenden Abgeordnetenwahlen von 1979: Lehr, Volker G.: Mexikos ,,reforma política“: Zur Erneuerung des mexikanischen Parteien- und Wahlsystems. In: Steger, Hanns-Albert, u. Jürgen Schneider (Hrsg.): Wirtschaft und gesellschaftliches Bewußtsein in Mexiko seit der Kolonialzeit. München 1980, S. 451-497 (Lateinamerika Studien, Bd. 6).

19 Diesen Trendextrapolationen liegen multiple Regressionsrechnungen zugrunde, in denenals unabhängige Variablen 1. Zeit und 2. der Umstand, ob die Abgeordnetenwahlen alleine oder zusammen mit Präsidentschaftswahlen abgehalten werden, fungieren. Die Varianz des PRI-Anteils wird zu 90,5\% aufgeklärt (signifikant auf dem 1-\%-Niveau), der Anteil der Oppositionsparteien zu 61,6 \% (signifikant auf dem 5-\%-Niveau) und der Nicht-Parteien-Anteil zu 93,4 \% (signifikant aú dem 1-\%-Niveau). 
darstellen würde, denn die Partei dient für viele ihrer Funktionsträger bloß als ein Vehikel zur Saturierung individueller Interessen. Der PRI und die in ihn inkorporierten Gewerkschaften und Bauernverbände könnten kaum noch auf ihre gegenwärtige Massenunterstützung rechnen, denn diese wird zu einem großen Teil unter Einsatz von Druckmitteln erzielt. Die Oppositionsparteien, deren reales Erstarken das PRI-Regime jahrelang mit allen Mitteln verhindert hat, wären für die Militärs gewiß kein Hindernis; unter Umständen könnten sie sogar mit der einen oder anderen Unterstützung aus den Reihen der Opposition rechnen.

-Wie Juan Linz feststellt, der sich seit Jahren mit autoritären Regimen befaßt, hat ,,der Zusammenbruch der meisten autoritären Regime nicht zur Errichtung einer Demokratie, sondern zur Einsetzung eines anderen autoritären Regimes geführt ${ }^{\text {‘20 }}$. Oft wurde die Möglichkeit einer politischen „Mexikanisierung“' der brasilianischen Militärherrschaft diskutiert ${ }^{21}$. Eine Diskussion in umgekehrter Richtung erscheint vielleicht angebrachter zu sein, auch wenn Staatspräsident López Portillo die Gefahr der ,,sudamericanización“ Mexikos, vor der er selber 1976 kurz vor seinem Amtsantritt gewarnt hatte ${ }^{22}$, in politischer Hinsicht bis auf weiteres abgewendet hat. Eine militärische Machtübernahme, in welcher Form auch immer, ist durchaus eine Möglichkeit, die man in Erwägung ziehen sollte, wenn man über Mexikos politische Zukunft nachdenkt.

20 Linz, Juan: The Breakdown of Democratic Regimes. Crisis, Breakdown and Reequilibration. Baltimore u. London 1978 , S. 7.

21 Siehe z. B. Stepan, Alfred (Hrsg.): Authoritarian Brazil. New Haven u. London 1973, passim.

22 Siehe Excélsior v. 27. 11. 1976. 
Tabelle 1

Personelle Stärke des mexikanischen Militärs im lateinamerikanischen Vergleich (1977)

\begin{tabular}{|c|c|c|c|}
\hline \multirow{3}{*}{ Lateinamerika } & \multirow{2}{*}{$\begin{array}{c}\text { Typ der } \\
\text { Regierung } \\
1977\end{array}$} & \multicolumn{2}{|c|}{ Personelle Stärke des Militärs } \\
\hline & & $\begin{array}{c}\text { Soldaten } \\
\text { auf } 1000 \mathrm{EW}\end{array}$ & $\begin{array}{l}\text { Soldaten } \\
\text { absolut }\end{array}$ \\
\hline & & 4,27 & 1440000 \\
\hline 1. Kuba & (Zivil) & 20,83 & 200000 \\
\hline 2. Chile & Militär & 10,57 & 111000 \\
\hline 3. Uruguay & Militär & 9,72 & 28000 \\
\hline 4. Guyana & Zivil & 8,73 & 7000 \\
\hline 5. Peru & Militär & 7,93 & 125000 \\
\hline 6. Argentinien & Militär & 5,92 & 155000 \\
\hline 7. Paraguay & (Zivil) & 5,03 & 15000 \\
\hline 8. Panama & Militär & 4,55 & 8000 \\
\hline 9. Ekuador & Militär & 4,10 & 30000 \\
\hline 10. Bolivien & Militär & 4,04 & 20000 \\
\hline 11. Venezuela & Zivil & 4,04 & 55000 \\
\hline 12. Brasilien & Militär & 3,81 & 450000 \\
\hline 13. Dominik. Rep. & Zivil & 3,62 & 19000 \\
\hline 14. Honduras & Militär & 3,53 & 12000 \\
\hline 15. Surinam & Zivil & 2,62 & 1000 \\
\hline 16. Nicaragua & (Zivil) & 2,56 & 6000 \\
\hline 17. Kolumbien & Zivil & 2,40 & 60000 \\
\hline 18. Guatemala & Militär & 2,17 & 14000 \\
\hline 19. El Salvador & Militär & 1,82 & 8000 \\
\hline 20. MEXIKO & Zivil & 1,57 & 100000 \\
\hline 21. Costa Rica*) & Zivil & 1,45 & 3000 \\
\hline 22. Haiti & (Zivil) & 1,29 & 7000 \\
\hline 23. Trinidad/Tobago & Zivil & 1,09 & 1000 \\
\hline 24. Barbados & Zivil & 0,74 & $(220)$ \\
\hline 25. Jamaika & Zivil & 0,46 & 1000 \\
\hline
\end{tabular}

Quelle: U. S. Arms Control and Disarmament Agency: World Military Expenditures and Arms Transfers 1968-1977. Washington D. C. 1979, Tab. II, S. 70-112. - Die Einteilung der Regierungen in Zivil- und Militärregierungen wurde von mir vorgenommen. ,(Zivil)“ bedeutet, daß man diese Regierungen (1977) nur mit Einschränkungen als zivil betrachten kann. 1980 (Stand September) wurden Ekuador, Panama und Peru wieder zivil regiert, Surinam hingegen militärisch.

\footnotetext{
*) Bürgerwehr (Guardia Civil)
} 
Tabelle 2

Mexikanische Militärausgaben im lateinamerikanischen Vergleich (1977)

\begin{tabular}{|c|c|c|c|}
\hline & $\begin{array}{c}\text { Typ der } \\
\text { Regierung } \\
1977\end{array}$ & $\begin{array}{c}\text { Militär } \\
\text { in \% } \\
\text { des BSP }\end{array}$ & $\begin{array}{l}1977 \\
\text { in Mill. } \\
\text { Dollar }\end{array}$ \\
\hline Lateinamerika & & 1,50 & 5710 \\
\hline 1. Peru & Militär & 6,33 & 887 \\
\hline 2. Kuba (1975) & (Zivil) & 6,16 & 389 \\
\hline 3. Guyana & Zivil & 3,10 & 13 \\
\hline 4. Chile & Militär & 2,29 & 261 \\
\hline 5. Uruguay & Militär & 2,05 & 80 \\
\hline 6. Argentinien & Militär & 2,04 & 761 \\
\hline 7. Bolivien & Militär & 1,96 & 62 \\
\hline 8. Nicaragua & (Zivil) & 1,93 & 38 \\
\hline 9. Ekuador & Militär & 1,90 & 110 \\
\hline 10. Honduras & Militär & 1,79 & 24 \\
\hline 11. Venezuela & Zivil & 1,74 & 622 \\
\hline 12. Dominik. Rep. & Zivil & 1,58 & 66 \\
\hline 13. Paraguay & (Zivil) & 1,39 & 28 \\
\hline 14. Brasilien & Militär & 1,03 & 1620 \\
\hline 15. Haiti & (Zivil) & 1,01 & 11 \\
\hline 16. El Salvador & Militär & 0,96 & 23 \\
\hline 17. Jamaika & Zivil & 0,92 & 22 \\
\hline 18. Guatemala & Militär & 0,77 & 38 \\
\hline 19. MEXIKO & Zivil & 0,73 & 515 \\
\hline 20. Kolumbien & Zivil & 0,70 & 117 \\
\hline 21. Panama & Militär & 0,70 & 15 \\
\hline 22. Trinidad/Tobago & Zivil & 0,32 & 8 \\
\hline 23. Barbados & Zivil & 0,24 & 1 \\
\hline 24. Costa Rica & Zivil & 0,00 & 0 \\
\hline --Surinam & Zivil & - & - \\
\hline
\end{tabular}

Quelle: U. S. Arms Control and Disarmament Agency: World Military Expenditures and Arms Transfers $1968-\cdots 1977$. Washington D. C. 1979, Tab. I, S. 27-69. 


\section{The Mexican Military: A Deviant Case in Latin America? \\ By VolKer LeHr}

It is quite normal in Latin America for the military to dictate the rules of politics. Mexico where the Partido Revolucionario Institucional (PRI) has governed in an authoritarian way for more than five decades - is considered to be one of the few exceptions. In terms of numbers and expenditure the Mexican armed forces are relatively small in comparison with other Latin American states. In the process of consolidation of the regime, which resulted from the popular revolution 1910-1917, the military was politically neutralized by a system of "divide et impera"'-measures and by economic as well as social benefits. Since 1946 all Mexican presidents have been civilians. However, all security units are controlled by the military. The main task of the Mexican armed forces is the maintenance of internal law and order. Particulary in rural areas there is a strong military control.

In recent years a clandestine "Brigada Blanca" has emerged, which had been formed by military personnel for the oppression of subversive activities. This brigade acts outside all constitutional norms and the lawful procedures. In this context, dangerous tendencies towards political dissociation cannot be overlooked. There are circumstances which do not make obligatory a return of the military to the power, but the potential is there. The early generations of officers, which identified themselves with the regime due to their own participation in the armed revolution, have now become extinct. The new cadres compare their position with that of their Latin American counterparts.

Social tensions are likely to increase in view of the unbalanced development of the country. The electoral results already show a marked loss of legitimacy of the ruling system. The growing intensity of the socio-economic crises and a persistent or even extending vacuum of legitimacy could lead to a situation, in which the military would no longer be willing to shore up a PRI-regime associated with corruption, graft and administrative inefficiency, but rather exercise power on its own term and responsibility and thus guarantee order and stability. 